\title{
Effects of preoxygenation with the three minutes tidal volume breathing technique in the elderly
}

\author{
Hyoseok Kang, Hye Jin Park, Seung Kwon Baek, Juyoun Choi, and So Jin Park \\ Department of Anesthesiology and Pain Medicine, Eulji General Hospital, College of Medicine, Eulji University, Seoul, Korea
}

Background: Preoxygenation with tidal volume breathing for $3 \mathrm{~min}$ is commonly used technique. An end tidal expiratory oxygen concentration greater than $90 \%$ is considerd to be adequate for preoxygenation. The aim of this study was to check the effects of preoxygenation on elderly patients through the comparison with young patients during the 3 min tidal volume breathing technique.

Methods: Sixty patients from ASA class I or II who were scheduled for elective orthopedic general surgery were divided into an elderly ( $>65 \mathrm{yr}$ ) group and a control (25-65 yr) group. Patients were instructed in the technique of preoxygenation. Preoxygenation was accomplished with an appropriately sized face mask connected to an anesthesia machine with $100 \%$ oxygen during 3 min with patients in both groups. Expired $\mathrm{O}_{2}, \mathrm{CO}_{2}$ concentration and oxygen saturation were recorded simultaneously for $3 \mathrm{~min}$.

Results: The elderly group showed significantly lower end tidal oxygen concentration than the control group from 50 sec to the end of study $(180 \mathrm{sec})$ with the 3 min tidal volume breathing technique $(\mathrm{P}<0.05)$. In $180 \mathrm{sec}$, the control group had over $90 \%$ (91.5\%) end tidal oxygen concentration, but in the elderly group end tidal oxygen concentration could not reach $90 \%$ (86.2\%). In oxygen saturation, the elderly group showed a significantly lower level until 40 sec from the start of study, and then two groups showed a similar levels of oxygen saturation until the end of study.

Conclusions: End tidal oxygen concentration did not reach $90 \%$ in $180 \mathrm{sec}$ in the elderly group during preoxygenation with the 3 min tidal volume breathing technique. (Korean J Anesthesiol 2010; 58: 369-373)

Key Words: Elderly, Mask, Oxygen concentration, Preoxygenation, 3 min tidal volume breathing.

\footnotetext{
Received: January 15, 2010. Revised: 1st, January 30, 2010; 2nd, February 10, 2009. Accepted: March 12, 2010.

Corresponding author: Hye Jin Park, M.D., Department of Anesthesiology and Pain Medicine, Eulji General Hospital, College of Medicine, Eulji University, Hagye-dong, Nowon-gu, Seoul 139-230, Korea. Tel: 82-2-970-8084, Fax: 82-2-970-8084, E-mail: anesthjin@hanmail.com The 85th Annual Scientific Meeting of Korean Society of Anesthesiologists, 2008, Seoul, Korea.

(c) This is an open-access article distributed under the terms of the Creative Commons Attribution Non-Commercial License (http:// creativecommons.org/licenses/by-nc/3.0/), which permits unrestricted non-commercial use, distribution, and reproduction in any medium, provided the original work is properly cited.
} 


\section{Introduction}

Preoxygenating with $100 \%$ oxygen before induction of general anesthesia and delaying the onset of arterial hemoglobin desaturation during subsequent apnea is standard practice in many institutions. Maximal preoxygenation is achieved when the alveolar, arterial, tissue, and venous compartment are all filled with oxygen. Hence, if most of the nitrogen in the lung is replaced by oxygen, end tidal expiratory oxygen concentration is reached over $90 \%$ as the result of adequate preoxgenation [1]. However, patients with a decreased capacity of oxygen loading or an increased oxygen extraction, or both, are desaturated during apnea much faster than healthy patients [2]. In elderly people total lung capacity is relatively unchanged by the age, but functional residual capacity falls below closing capacity which increases with age. The change in the relationship between functional residual capacity and closing capacity causes an increased ventilation perfusion mismatch, which leads to a lower oxygen stores than in young adults [3].

Various techniques and regimens have been advocated to accomplish preoxygenation, and several studies have demonstrated that most subjects are optimally oxygenated after 3 min of normal tidal volume breathing of $100 \%$ oxygen using the standard breathing systems $[1,4]$. Various endpoints such as minimum end tidal nitrogen fraction, maximum arterial oxygen tension, maximum end tidal oxygen concentration and time taken for hemoglobin to desaturate to a certain level after anesthesia induced apnea have been used [5-7]. Although these various end points are commonly used for evaluating the effect of preoxygenation, end tidal oxygen concentration has been favored to determine the end point for preoxygenation as it measures the alveolar gas concentration, and thus more accurately determines the degree of denitrogenation $[8,9]$.

In this study we checked the effect of preoxgenation in elderly patients through the comparison with young patients by using end tidal oxygen concentration monitoring and pulse oxymetry as an end point for oxygenation during the 3 min tidal volume breathing technique.

\section{Materials and Methods}

The study was approved by the hospital ethical committee, and written informed consent was obtained from all the patients. Sixty ASA physical status 1 or 2 patients presenting for elective general and orthopedic surgery were divided into two groups: Control group comprised 30 adult patients whose age ranged from 25 to $65 \mathrm{yr}$ and elderly group comprised 30 elderly patients aged $65 \mathrm{yr}$ above. During the preoperative assessment the method of preoxygenation was explained to the patients. Patients with clinically significant cardiovascular, respiratory, neurologic, or endocrine disease, and obesity (BMI $>30$ ) or those for whom difficulties were anticipated in mask ventilation were excluded.

Patients were brought, unpremedicated, to the operating room, and then the patients were again instructed in the technique of preoxygenation. Ample time was allowed for patients to become familiar with mask breathing before applying a tight seal. This study tested how the mask fitting is adequate by checking the tidal volume and wave of capnograph with medical air through the anesthesia machine. A single anesthesia machine (Model; Avance, Datex-Ohmeda, Madison, USA) with a circle system was used throughout the study. Monitoring was done by an automated blood pressure device, electrocardiogram, and gas monitor (Airway module, Datex-Ohmeda, Madison, USA) which recorded inspired and expired $\mathrm{O}_{2}, \mathrm{CO}_{2}$ concentrations and a pulse oximeter (Philips V24E, Germany) was applied to the patient's finger.

For baseline measurements, patients breathed room air with the tidal volume breathing technique for $3 \mathrm{~min}$ to record $\mathrm{O}_{2}, \mathrm{CO}_{2}$ concentration and oxygen saturation in the supine position. Afterwards the face mask was applied firmly to patient's face in order to prevent air leakages, but not so tightly as to cause discomfort. Before preoxygenation, the tidal volume was checked over $6 \mathrm{ml} / \mathrm{kg}$ and end tidal $\mathrm{CO}_{2}$ concentration was checked by using $8 \mathrm{~L} / \mathrm{min}$ flow of medical air under the circle system. By pushing the button of oxygen instead of medical air, preoxygenation was accomplished. Preoxygenation was done with an appropriately sized clear face mask connected to $100 \%$ oxygen filled anesthesia machine. The face mask was applied to the patients in the supine position in both groups. Oxygen flow rate was set at $8 \mathrm{~L} / \mathrm{min}$, and patients continued to breathe normally at tidal volumes for $3 \mathrm{~min}$. Expired $\mathrm{O}_{2}$ and $\mathrm{CO}_{2}$ concentration and oxygen saturation were recorded simultaneously every $10 \mathrm{sec}$ for first $1 \mathrm{~min}$ and then every $20 \mathrm{sec}$ for the following $2 \mathrm{~min}$ in both groups.

Statistical analysis was performed using GraphPad Prism ${ }^{\circledR}$ (version 5.0, GraphPad Software, Inc. USA). Values are reported as mean $\pm \mathrm{SD}$. The demographic data was compared using $t$-test. The comparison of end tidal oxygen and $\mathrm{CO}_{2}$ concentration, and oxygen saturation between two groups were analyzed by using a two way repeated measure ANOVA. $P$ values of less than 0.05 were considered to be statistically significant.

\section{Results}

The demographic data for two groups are shown in Table 1. The mean age of the elderly group was 74.8 years and the control group was 42.2 years. Although body weight and height in the control group $(65.6 \mathrm{~kg}$ and $165.1 \mathrm{~cm})$ were greater than the elderly group's (57.5 kg and $156.9 \mathrm{~cm}$ ), there was no significant 
Table 1. Characteristics of Patients

\begin{tabular}{lcc}
\hline \multicolumn{1}{c}{ Variables } & Control group $(\mathrm{n}=30)$ & Elderly group $(\mathrm{n}=30)$ \\
\hline Age $(\mathrm{yr})$ & $42.2 \pm 2.3$ & $74.8 \pm 1.4$ \\
$\mathrm{Wt}(\mathrm{kg})$ & $65.6 \pm 2.3$ & $57.5 \pm 1.8$ \\
$\mathrm{Ht}(\mathrm{cm})$ & $165.1 \pm 1.3$ & $156.9 \pm 1.7$ \\
$\mathrm{BMI}$ & $24.0 \pm 0.7$ & $23.4 \pm 0.7$ \\
$\mathrm{Hb}(\mathrm{g} / \mathrm{dl})$ & $13.5 \pm 0.3$ & $12.9 \pm 0.3$ \\
$\mathrm{Sex}(\mathrm{M} / \mathrm{F})$ & $14 / 16$ & $12 / 18$ \\
\hline
\end{tabular}

Values are presented mean \pm SD or number of patients. Control group: $25-65 \mathrm{yr}$, Elderly group: over $65 \mathrm{yr}$, BMI: body mass index.

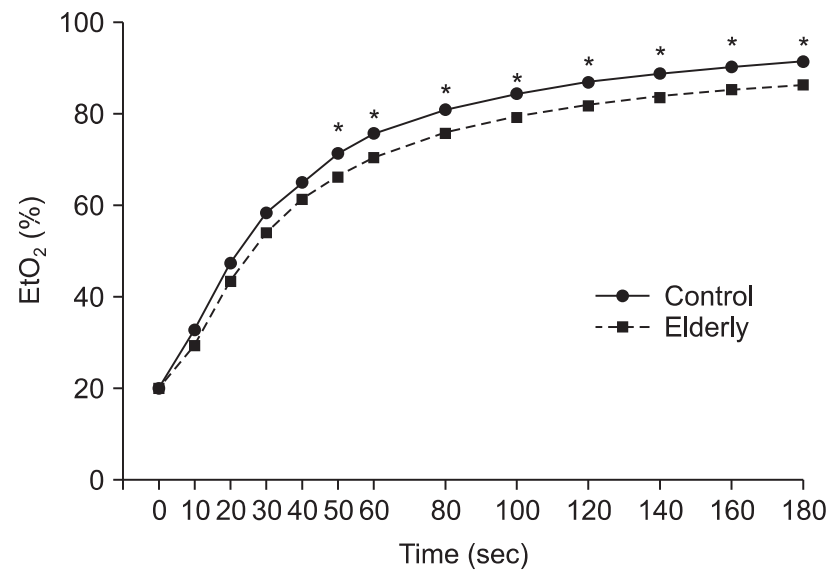

Fig. 1. Comparison of control (25-65 yr) and elderly ( $>65 \mathrm{yr}$ ) group on end tidal oxygen concentration with the 3 min tidal volume breathing technique. Elderly group shows significantly lower end tidal oxygen concentration than control group from $50 \mathrm{sec}$ to $180 \mathrm{sec}$. $\mathrm{EtO}_{2}$ : end tidal oxygen concentration, ${ }^{*} \mathrm{P}<0.05$ compared to control group.

difference shown in the BMI (Body Mass Index). There were no statistically significant differences between the two groups in hemoglobin level and the male to female ratio.

End tidal oxygen concentration results are shown in Fig. 1. There were significant differences between two groups in end tidal oxygen concentration from $50 \mathrm{sec}$ to the end of study (180 sec) with the 3 min tidal volume breathing technique. In $180 \mathrm{sec}$, the control group had over 90\% (91.5\%) end tidal oxygen concentration, but in the elderly group end tidal oxygen concentration could not reach $90 \%$ (86.2\%). Patients in the elderly group had a significantly lower oxygen saturation compared to the control group when they were breathing room air and the lower leveled oxygen saturation was continued until $40 \mathrm{sec}$ from the start of study with $100 \%$ oxygen breathing. However after $40 \mathrm{sec}$, two groups showed similar levels of oxygen saturation until the end of the study (Fig. 2). There was no significant difference between the two groups in end tidal $\mathrm{CO}_{2}$ concentration with the tidal volume breathing technique (Fig. 3).

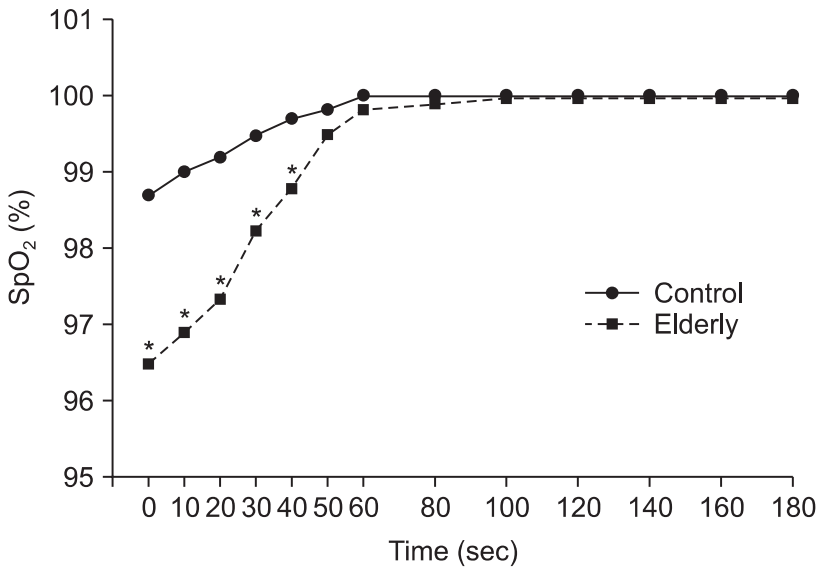

Fig. 2. Comparison of control (25-65 yr) and elderly ( $>65$ yr) group on oxygen saturation with the tidal volume breathing technique. Elderly group shows significantly lower oxygen saturation than control group until $40 \mathrm{sec}$. $\mathrm{SpO}_{2}$ : oxygen saturation by pulse oximetry, $* \mathrm{P}<0.001$ compared to control group.

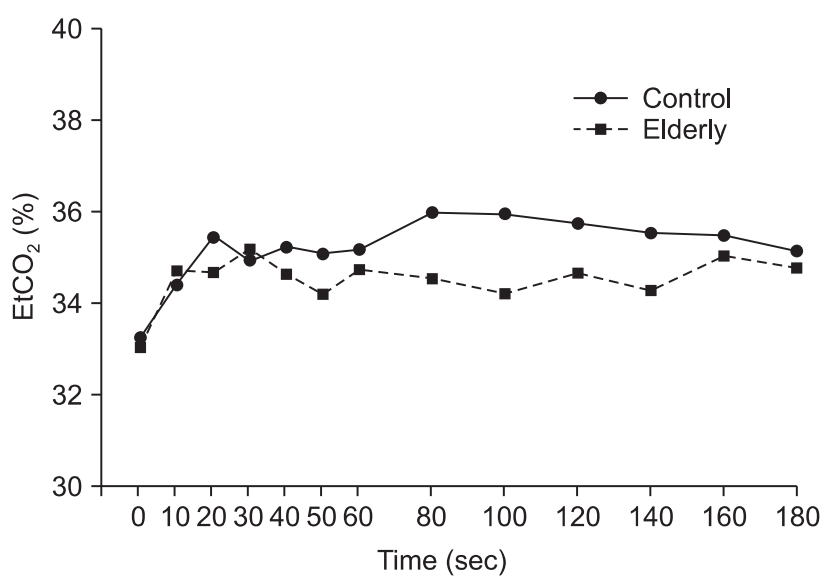

Fig. 3. Comparison of control (25-65 yr) and elderly ( $>65 \mathrm{yr}$ ) group on end tidal $\mathrm{CO}_{2}$ concentration with the tidal volume breathing technique. There is no significant difference between the groups. $\mathrm{EtCO}_{2}$ : end tidal carbon dioxide concentration.

\section{Discussion}

Preoxygenation depends on the spontaneous breathing of $100 \%$ oxygen, which denitrogenates the functional residual capacity (FRC) of the lungs. Hence preoxygenation increases the FRC oxygen store and delays the onset of arterial desaturation and hypoxemia during the apneic period following the induction of anesthesia. Various methods of preoxygenation to protect against hypoxemia after the induction of anesthesia have been recommended. Tidal volume breathing of $100 \%$ oxygen for $3 \mathrm{~min}$ has been commonly used, and four deep vital capacity breaths of oxygen taken within $0.5 \mathrm{~min}$, as well as eight deep vital capacity breaths of oxygen taken within 1 min are 
also used $[5,6,10]$. However, although they show similar peak arterial oxygen saturation values, it is known that the 3 min tidal volume breathing technique gives more reliable protection against desaturation during apnea than the four maximal breath technique after preoxygenation $[11,12]$. Some investigations showed that 3 min tidal volume breathing was equally effective in increasing arterial oxygen tension as four deep breath technique $[6,13,14]$. This similarity leads to the conclusion that four maximal breathing method provides the same amount of preoxygenation as 3 min tidal volume breathing method. However, other studies have shown that patients preoxygenated using four maximal breathing method desaturate faster than patients preoxygenated using the 3 min tidal volume breathing method $[11,12,15]$. It means 3 min tidal volume breathing provided more effective preoxygenation, and longer protection against hypoxemia during apnea than four deep breathing technique. In elderly patients, Valentine et al. [11] showed that preoxygenation with 3 min tidal volume breathing of $100 \%$ oxygen offers more protection against hypoxia due to prolonged apnea after induction of anesthesia than does four maximal breaths of $100 \%$ oxygen. The same results were shown in other many previous studies which were based on mainly younger patients or healthy volunteers.

Maximal preoxygenation is achieved when the alveolar, vascular, and tissue compartments are all saturated with oxygen [16,17]. End points of alveolar preoxygenation and denitrogenation have been defined as an end tidal oxygen concentration of approximately $90 \%$ and an end tidal nitrogen concentration of $5 \%$. However, failure to breathe a high oxygen concentration, inadequate time for preoxygenation, and the presence of a leak under the mask can result in submaximal alveolar oxygenation $[4,16]$. In this study end tidal oxygen concentration in the elderly was $86 \%$ after 3 min tidal volume breathing with $100 \%$ oxygen and $90 \%$ was not reached, as we expected, but in the control group, end tidal oxygen concentration was $90 \%$ in $160 \mathrm{sec}$ after preoxygenation. Although oxygen saturation in the elderly reached $99 \%$ around $40 \mathrm{sec}$ with rapid increasing in preoxygenation, end tidal oxygen concentration was $61 \%$ in $40 \mathrm{sec}$, and it was lower than the control group, at $65 \%$. It showed that end-tidal oxygen concentration monitoring is a more reliable guide for adequate preoxygenation than pulse oximetry which is more commonly used in anesthesia. Bhatia et al. [1] reported that elderly group took 2.74 min of mean time to achieve an end tidal oxygen concentration of $90 \%$, the other side, our elderly group did not reach $90 \%$ within 3 min. Because the mean age of the elderly group in this study was 74.8 years, it is older than Bhatia's study group with the mean age of 62.5 years. The mean age difference between the two studies is over 10 years. Therefore our findings suggest that the elderly group over 70 years of age is different in pulmonary function from the young group, leading to lower end tidal oxygen concentration during preoxygenation.

The functional changes in the lung with aging include increased anatomic dead space, decreased diffusing capacity, and increased closing capacity lead to impaired gas exchange. Although total lung capacity is relatively unchanged by the age, the residual volume increases by $5 \%$ to $10 \%$ per decade and functional residual capacity falls below the closing capacity which increases with age [3]. The change in the relationship between functional residual capacity and closing capacity cause an increased ventilation-perfusion mismatch. These alterations in the respiratory system of the elderly thus appears to show more fluent curve in the end tidal oxygen concentration than that of young people and the elderly need more time to reach $90 \%$ of end tidal oxygen concentration in preoxygenation (Fig. 1).

The other reason why the end tidal oxygen concentration did not reached $90 \%$ in elderly group is thought to be due to the degree of mask fitting to the face. Most studies about preoxygenation have used the method of a tight fitting mask to prevent air leakage during preoxygenation. However, effective preoxygenation sometimes failed due to facial hair or a poor mask fit [1]. In fact, anesthesiologists often report that the mask represents a significant discomfort to the patients and many patients find preoxygenation objectionable. According to the study of patient discomfort caused by the oxygen mask during preoxygenation [18], the patient expected a moderate discomfort caused by the mask prior to the operation, whereas postoperatively, the patient mentioned no discomfort. An overestimation of the average patient discomfort by the anesthesiologist may contribute to the reluctance of using routine preoxygenation. We have experienced that the patients for this study did not mentioned significant discomfort from the mask or difficulty breathing. However, it was difficult to check that the mask on the patient's face was adequate to prevent the air leakage without tightness. Another problem we found during preoxygenation was teeth that were partial or in a total edentate state in the elderly group. The edentate state made the mask difficult to fit the face because of sunken cheeks. During the study we tried to prevent air leakage through the mask and continuously checked the adequate tidal volume in the monitor.

In summary, the increasing rate of end tidal oxygen concentration in the elderly group during preoxygenation is slower than that of the young group, so $90 \%$ is not reached in $180 \mathrm{sec}$ with the 3 min tidal volume breathing technique. Hence, if there is no appreciable air leakage under the mask, the elderly group needs more time than $180 \mathrm{sec}$ to achieve $90 \%$ of end tidal oxygen concentration during preoxygenation. 


\section{References}

1. Bhatia PK, Bhandari SC, Tulsiani KL, Kumar Y. End-tidal oxygraphy and safe duration of apnoea in young adults and elderly patients. Anaesthesia 1997; 52: 175-8.

2. Farmery AD, Roe PG. A model to describe the rate of oxyhaemoglobin desaturation during apnoea. Br J Anaesth 1996; 76: 284-91.

3. Frederick Sieber RP. Geriatric Anesthesia. In: Miller's Anesthesia. 7th ed. Edited by Miller RD, Fleisher LA, Wiener-Kronish JP, Young WL: Philadelphia, Churchill Livingstone. 2009, pp 2263-4.

4. Berthoud M, Read DH, Norman J. Pre-oxygenation--how long? Anaesthesia 1983; 38: 96-102.

5. Baraka AS, Taha SK, Aouad MT, El-Khatib MF, Kawkabani NI. Preoxygenation: comparison of maximal breathing and tidal volume breathing techniques. Anesthesiology 1999; 91: 612-6.

6. Gold MI, Duarte I, Muravchick S. Arterial oxygenation in conscious patients after 5 minutes and after 30 seconds of oxygen breathing. Anesth Analg 1981; 60: 313-5.

7. Winship S, Skinner A. Vital capacity and tidal volume preoxygenation with a mouthpiece. Br J Anaesth 1998; 81: 787-9.

8. Pandit JJ, Duncan T, Robbins PA. Total oxygen uptake with two maximal breathing techniques and the tidal volume breathing technique: a physiologic study of preoxygenation. Anesthesiology 2003; 99: 841-6.

9. Edmark L, Kostova-Aherdan K, Enlund M, Hedenstierna G. Optimal oxygen concentration during induction of general anesthesia. Anesthesiology 2003; 98: 28-33.

10. Gold MI. Preoxygenation. Br J Anaesth 1989; 62: 241-2.

11. Valentine SJ, Marjot R, Monk CR. Preoxygenation in the elderly: a comparison of the four-maximal-breath and three-minute techniques. Anesth Analg 1990; 71: 516-9.

12. Gambee AM, Hertzka RE, Fisher DM. Preoxygenation techniques: comparison of three minutes and four breaths. Anesth Analg 1987; 66: 468-70.

13. Norris MC, Dewan DM. Preoxygenation for cesarean section: a comparison of two techniques. Anesthesiology 1985; 62: 827-9.

14. Goldberg ME, Norris MC, Larijani GE, Marr AT, Seltzer JL. Preoxygenation in the morbidly obese: a comparison of two techniques. Anesth Analg 1989; 68: 520-2.

15. McCarthy G, Elliott P, Mirakhur RK, McLoughlin C. A comparison of different pre-oxygenation techniques in the elderly. Anaesthesia 1991; 46: 824-7.

16. Benumof JL. Preoxygenation: best method for both efficacy and efficiency. Anesthesiology 1999; 91: 603-5.

17. Campbell IT, Beatty PC. Monitoring preoxygenation. Br J Anaesth 1994; 72: 3-4.

18. Schlack W, Heck Z, Lorenz C. Mask tolerance and preoxygenation: a problem for anesthesiologists but not for patients. Anesthesiology 2001; 94: 546. 